\title{
Use of Pyrosequencing and Denaturing Gradient Gel Electrophoresis to Examine the Effects of Probiotics and Essential Oil Blends on Digestive Microflora in Broilers Under Mixed Eimeria Infection
}

\author{
Michael E. Hume, Nei A. Barbosa,,3 Scot E. Dowd, ${ }^{3}$ Nilva K. Sakomura, ${ }^{2}$ Armen G. Nalian, ${ }^{4}$ \\ Alexandra Martynova-Van Kley, and Edgar O. Oviedo-Rondón ${ }^{5}$
}

\begin{abstract}
A protective digestive microflora helps prevent and reduce broiler infection and colonization by enteropathogens. In the current experiment, broilers fed diets supplemented with probiotics and essential oil (EO) blends were infected with a standard mixed Eimeria spp. to determine effects of performance enhancers on ileal and cecal microbial communities (MCs). Eight treatment groups included four controls (uninfected-unmedicated [UU], unmedicated-infected, the antibiotic BMD plus the ionophore Coban as positive control, and the ionophore as negative control), and four treatments (probiotics BC-30 and Calsporin; and EO, Crina Poultry Plus, and Crina PoultryAF). Day-old broilers were raised to 14 days in floor pens on used litter and then were moved to Petersime batteries and inoculated at 15 days with mixed Eimeria spp. Ileal and cecal samples were collected at 14 days and 7 days postinfection. Digesta DNA was subjected to pyrosequencing for sequencing of individual cecal bacteria and denaturing gradient gel electrophoresis (DGGE) for determination of changes in ileal and cecal MC according to percentage similarity coefficient (\%SC). Pyrosequencing is very sensitive detecting shifts in individual bacterial sequences, whereas DGGE is able to detect gross shifts in entire MC. These combined techniques offer versatility toward identifying feed additive and mild Eimeria infection modulation of broiler MC. Pyrosequencing detected 147 bacterial species sequences. Additionally, pyrosequencing revealed the presence of relatively low levels of the potential human enteropathogens Campylobacter sp. and four Shigella spp. as well as the potential poultry pathogen Clostridiun perfringens. Pre- and postinfection changes in ileal (56\%SC) and cecal $(78.5 \%$ SC) DGGE profiles resulted from the coccidia infection and with increased broiler age. Probiotics and EO changed MC from those seen in UU ilea and ceca. Results potentially reflect the performance enhancement above expectations in comparison to broilers not given the probiotics or the specific EO blends as feed supplements.
\end{abstract}

\section{Introduction}

D IGESTIVE MICROBIAL BACTERIA are gaining greater recognition as pivotal components in promoting and maintaining broiler vitality and production. Conventional dietary strategies have included antibiotic growth promoters (AGP) as modifiers of digestive microbial communities (MCs) to improve livestock production (Jukes et al., 1950; Jukes,
1955; Dibner and Richards, 2005). Low-dosage applications of AGP are thought to limit low-level enteropathogen infections and decrease populations of bacteria that produce substances harmful to the host (Jukes, 1955). Current trends are increasingly moving toward the development of AGP alternatives to positively impact digestive MC. More recent dietary strategies recognize plant essential oil (EO) extracts as modifiers and supporters of MC (Kim et al., 1995; Jamroz et al., 2005;

\footnotetext{
${ }^{1}$ Food and Feed Safety Research Unit, Southern Plains Agricultural Research Center, Agricultural Research Service, U.S. Department of Agriculture, College Station, Texas.

${ }^{2}$ FMVZ, Universidade Estadual Paulista (UNESP), Jaboticabal, Brazil.

${ }^{3}$ Research and Testing Laboratories, Medical Biofilm Research Institute, Lubbock, Texas.

${ }^{4}$ Stephen F. Austin State University, Nacogdoches, Texas.

${ }^{5}$ North Carolina State University, Raleigh, North Carolina.
} 
Peñalver et al., 2005; Hume et al., 2006; Oviedo-Rondón et al., 2006; Jung et al., 2008). These EO phytotherapeutics may function as substrates for beneficial bacteria, adversely affect susceptible bacteria, or promote the growth of bacteria that restrict the growth of bacteria harmful to host (Hume et al., 2006; Oviedo-Rondón et al., 2006; Si et al., 2006; Jung et al., 2008).

Supplementing young chicks with beneficial bacteria enhance early establishment of a protective MC and live performance (Nurmi and Rantala, 1973; Lloyd et al. 1977; Barnes et al., 1980). The growing recognition of the importance of healthy MC has resulted in increased interests in bacterial probiotic dietary supplements (Blankenship et al., 1993; Nisbet et al., 1993; Schneitz et al., 1998; Flint and Garner, 2009). Probiotic supplements offer early protection against enteropathogen invasion and colonization, can stimulate immune response, and may take advantage of nutritive components unavailable to the host.

In the current report, the effects of two commercial probiotics and two commercial specific EO on ileal and cecal MC in broiler chickens were evaluated before and after a mixed Eimeria spp. infection. Two molecular techniques, pyrosequencing and denaturing gradient gel electrophoresis (DGGE), were applied to monitor the impact of treatments and Eimeria infection on MC. The combined analyses of the two techniques were applied to give views at two levels of community changes. Pyrosequencing was applied to examine the impact of treatment on pre- and postinfection cecal on individual bacteria. DGGE was used for more gross comparisons of treatments on pre- and postinfected ileal and cecal MC.

\section{Materials and Methods}

\section{Broiler husbandry}

Day-old male Ross 708 chickens (384) were randomly placed in 48 floor pens (8/pen), each with litter used by broilers given the same treatments in two previous experiments. Litter management was applied to simulate commercial production systems. Downtime between experiments was 12 days. No additional bedding material was added. Pens were distributed to have an empty pen without chickens and without litter between pens to avoid cross contamination. Litter management between flocks was done with the same tools in all pens of similar treatment, and equipment was cleaned and disinfected before handling litter in pens assigned to different treatments. Daily biosecurity measure- ments were taken to avoid cross contamination among pens or cages. From 1 to 14 days, personnel did not enter the pens. Personnel did not have to enter pens for routine management, and disposable boots and gloves were used for pens, equipment, birds, feed, and samples assigned different treatments.

Chickens were distributed in eight treatments with six replicates each. Broilers were given balanced feed (NRC, 1994) and water provided ad libitum. At 14 days of age, 288 broilers (36/treatment) were transferred to Petersime batteries (6/ cage). Lighting simulated commercial conditions: 1 to 8 days, $23 \mathrm{~h}$ of light/day at 2.0 foot-candles, $1 \mathrm{~h}$ of darkness between 2 and $3 \mathrm{am}$; and 8 to 22 days, $6 \mathrm{~h}$ of darkness between 9:00 pm and 3:00 am. Rearing was according to safe practices authorized by the Animal Use Protocol Committee.

\section{Diets and treatments}

The eight treatments (Table 1) included four controls and four treatment diets. Broilers were fed starter crumbles to 22 days of age. All diets contained the phytase enzyme Ronozyme P CT (DSM Nutritional Products, Basal, Switzerland) at $168 \mathrm{~g} / \mathrm{ton}$. Diets also contained vermiculite as filler to allow replacement by either phytase, or enough dicalcium phosphate to add $0.1 \%$ phosphorus in treatment Crina Poultry AF 100 ppm (CPF) (Table 1), or probiotics or the specific EO blend according to treatment distribution.

\section{Coccidia}

All groups except uninfected-unmedicated control (UU) were given at 15 days of age a standard oral mixed Eimeria inoculum of sporulated oocysts: E. acervulina $\left(2 \times 10^{5} / \mathrm{mL}\right)$, E. maxima $\left(2.5 \times 10^{4} / \mathrm{mL}\right)$, and E. tenella $\left(5 \times 10^{4} / \mathrm{mL}\right)$ (provided by Dr. Mark C. Jenkins, Animal Parasitic Diseases Laboratory, Agricultural Research Service, U.S. Department of Agriculture). Oocytes were susceptible to ionophore.

\section{Sample collection and DNA extraction}

Ileal and cecal digesta were collected from six broilers per group pre-Eimeria infection at 14 days of age and from six broilers per group postinfection at 22 days of age. Samples were lyophilized and stored at $-80^{\circ} \mathrm{C}$ until DNA extraction. Lyophilized samples $(0.25 \mathrm{~g})$ were suspended in $180 \mu \mathrm{L}$ of lysis buffer (QIAmp DNA Mini Kit; QIAgen Incorporated, Valencia, CA) containing $20 \mathrm{mg} / \mathrm{mL}$ of lysozyme and extracted according to kit instructions.

Table 1. Treatment Groups

\begin{tabular}{lllc}
\hline Treatment & \multicolumn{1}{c}{ Treatment description } & Eubiotic & Ionophore \\
\hline UU & Unmedicated-uninfected control & None & No \\
UI & Unmedicated-infected control & None & No \\
PC & Positive control & None & Yes \\
NC & Negative control & None & Yes \\
BC30 & BC-30 & Probiotic & Yes \\
CAL & Calsporin & Probiotic & No \\
CPP & Crina Poultry Plus $300 \mathrm{ppm}$ & Essential oil blend & No \\
CPF & Crina Poultry AF $100 \mathrm{ppm}$ & Essential oil blend & Yes \\
\end{tabular}

AGP, antibiotic growth promoter. 


\section{Pyrosequencing massively parallel bacterial tag-encoded FLX amplicon pyrosequencing}

Bacterial tag-encoded FLX amplicon pyrosequencing was performed as described previously using Gray28F 5'TTTGATCNTGGCTCAG and Gray519r 5'GTNTTACNG CGGCKGCTG (Bailey et al., 2010a,b; Callaway et al., 2010; Finegold et al., 2010; Gontcharova et al., 2010a,b; Olafson et al., 2010; Pitta et al., 2010; Smith et al., 2010; Stephenson et al., 2010; Williams et al., 2010; Andreotti et al., 2011; Handl et al., 2011; Ishak et al., 2011) with primers numbered relative to Escherichia coli $16 \mathrm{~S}$ rDNA. Initial generation of the sequencing library utilized a one-step polymerase chain reaction (PCR) with a total of 30 cycles, a mixture of HotStart and HotStar high fidelity taq polymerases (Qiagen), and amplicons originating and sequencing extending from the $28 \mathrm{~F}$ with average read length of $400 \mathrm{bp}$. Tag-encoded FLX amplicon pyrosequencing analyses utilized Roche 454 FLX instrument with Titanium reagents, titanium procedures performed at the Research and Testing Laboratory (Lubbock, TX) based upon RTL protocols (www.researchandtesting.com).

\section{Pyrosequence bacterial diversity data analysis}

After sequencing, all failed sequence reads, low quality sequence ends and tags, and primers were removed and sequence collections depleted of any nonbacterial ribosome sequences and chimeras using B2C2 (Gontcharova et al., 2010b) as previously described (Bailey et al., 2010a,b; Finegold et al., 2010; Gontcharova et al., 2010a; Olafson et al., 2010; Pitta et al., 2010; Smith et al., 2010; Stephenson et al., 2010; Williams et al., 2010; Andreotti et al., 2011; Handl et al., 2011; Ishak et al., 2011). To identity the remaining bacterial sequences, sequences were denoised, assembled into clusters, and queried using a distributed BLASTn .NET algorithm (Dowd et al., 2005) against a high-quality $16 \mathrm{~S}$ bacterial sequence database (based upon similar criteria utilized by RDP ver 9 (Cole et al., 2009)) derived and curated monthly from NCBI (01-01-11). Using a .NET and C\# analysis pipeline, the resulting BLASTn outputs were compiled, validated using taxonomic distance methods, and data reduction analysis performed (Bailey et al., 2010a,b; Callaway et al., 2010; Finegold et al., 2010; Gontcharova et al., 2010a,b; Olafson et al., 2010; Pitta et al., 2010; Smith et al., 2010; Stephenson et al., 2010; Williams et al., 2010; Andreotti et al., 2011; Handl et al., 2011; Ishak et al., 2011).

\section{Pyrosequence bacterial identification}

Based upon the above BLASTn-derived sequence identity (percent of total length query sequence that aligns with a given database sequence) and validated using taxonomic distance methods, the bacteria were classified at the appropriate taxonomic levels based upon the following criteria. Sequences with identity scores, to known or well-characterized $16 \mathrm{~S}$ sequences, $>97 \%$ identity ( $<3 \%$ divergence) were resolved at the species level, between $95 \%$ and $97 \%$ at the genus level, between $90 \%$ and $95 \%$ at the family, and between $85 \%$ and $90 \%$ at the order level, $80 \%$ and $85 \%$ at the class, and $77 \%$ to $80 \%$ at phyla. Evaluations presented at each taxonomic level, including percentage compilations, represent all sequences resolved to their primary identification or their closest relative (Bailey et al., 2010a,b; Callaway et al., 2010; Finegold et al., 2010; Gontcharova et al., 2010a,b; Olafson et al., 2010; Pitta et al., 2010; Smith et al., 2010; Stephenson et al., 2010; Williams et al., 2010; Andreotti et al., 2011; Handl et al., 2011; Ishak et al., 2011).

\section{Denaturing gradient gel electrophoresis}

Extracted DNA adjusted to $42 \mathrm{ng} / \mu \mathrm{L}$ and $1 \mu \mathrm{L}$ from each of six samples per treatment were pooled for PCR (Zhou et al., 2007), targeting the $16 S$ rDNA gene V3 region. Reactions were performed in $25 \mu \mathrm{L}$ according to Muyzer et al. (1993) with modification (Hume et al., 2003). Electrophoresis was conducted using a DCode Universal Mutation Detection System (Bio-Rad Laboratories, Hercules, CA). Band pattern relatedness was determined with Molecular Analysis Fingerprinting Software, version 1.6 (Bio-Rad Laboratories), based on the Dice similarity coefficient (SC) and unweighted pair group method using arithmetic averages (UPGMA) for clustering analysis and comparison according to percentage SC (\%SC).

\section{Statistical analysis}

The DGGE profiles were digitized and converted to a binary matrix using a script (available on request) written in the Python programming language (Python Software Foundation, Hampton, NH). Taxonomic assignment of pyrosequencing reads was carried out using BLAST. The annotated sequences were used to calculate the relative percent abundance of taxa per sample. The pyrosequencing abundance matrix and the DGGE binary matrix were further analyzed with the $\mathrm{R}$ statistical software (Foundation for Statistical Computing, Vienna, Austria; www.R-project.org) using various R-packages; the similarity matrix was computed with Sorensen's coefficient using the ade4 package (Chessel et al., 2004); clustering and principal component analysis (PCA) were carried out using the vegan package (Oksanen et al., 2010; Community ecology package, version 1.17). Clustering was performed using Bray-Curtis dissimilarity index and average linkage method (Oksanen et al., 2010). Correlation of body weights and factors such as treatment and infection with ordination was calculated using envfit and ordisurf functions available in vegan package. The significance of the correlation was evaluated with 10,000 permutations. Performance was analyzed by Tukey's test with a level of significance $p<0.05$.

\section{Results}

\section{Pyrosequencing}

Nine bacteria phyla were detected among the 48,461 sequences identified in cecal samples taken from eight treatment groups in Eimeria pre- and postinfection broilers: Actinobacteria, Bacteroidetes, Candidatus Poribacteria, Cyanobacteria, Firmicutes, Fusobacteria, Proteobacteria, Tenericutes, and Verrucomicrobia. Bacteroidetes, Firmicutes, and Proteobacteria are represented by at least $1 \%$ of identified sequences (Tables 2 and 3). Orders Bacteroidales, Clostridiales, Bacteriales, and Enterobacteriales were detected in preinfection ceca, whereas postinfection orders were Clostridiales, Bacteroidales, Campylobacterales, Lactobacillales, and Enterobacteriales. Further classification of sequences identified, including those at $<1 \%$, revealed 15 classes, 20 orders, 44 families, 90 genera, and 147 species.

Seven species representing at least $1 \%$ of sequences were detected at preinfection (Table 2), whereas 12 were detected at 
Table 2. Pyrosequenced Percentage Bacterial Species in Cecal Contents of Broilers at 14 Days of Age

\begin{tabular}{|c|c|c|c|c|c|c|c|c|}
\hline \multirow[b]{2}{*}{ Species } & \multicolumn{8}{|c|}{ Treatment } \\
\hline & UU & UI & $P C$ & $N C$ & BC30 & $C A L$ & $C P P$ & $C P F$ \\
\hline Bacteroides sp. & 1.1 & 0.9 & 0.3 & 0.6 & 11.7 & 2.9 & 4.2 & 0.3 \\
\hline Clostridium sp. & 28.4 & 17.6 & 25.4 & 10.3 & 5.6 & 4.9 & 7.6 & 11.4 \\
\hline Faecalibacterium sp. & 11.2 & 12.0 & 5.6 & 5.6 & 7.2 & 12.5 & 9.8 & 1.0 \\
\hline Lactobacillus sp. & 3.8 & 3.6 & 9.3 & 7.6 & 2.5 & 5.9 & 16.2 & 22.5 \\
\hline Papillibacter sp. & 13.6 & 10.4 & 8.6 & 11.3 & 12.2 & 16.7 & nd & 9.3 \\
\hline Roseburia & 3.2 & 10.1 & 6.3 & 7.9 & 5.6 & 4.1 & 5.8 & 9.7 \\
\hline Ruminococcus sp. & 8.1 & 12.0 & 13.2 & 16.6 & 8.0 & 8.9 & 18.7 & 13.8 \\
\hline Shigella sonnei & 0.3 & nd & nd & nd & nd & nd & nd & nd \\
\hline Percentage total & 69.7 & 66.6 & 68.8 & 59.9 & 52.8 & 55.8 & 62.3 & 68.0 \\
\hline
\end{tabular}

UU, unmedicated-uninfected control; UI, unmedicated-infected control; PC, positive control; NC, negative control; $\mathrm{BC} 30$, BC-30 probiotic; CAL, calsporin probiotic; CPP, Crina Poultry Plus 300 ppm; CPF, Crina Poultry AF 100 ppm; nd, not detected.

postinfection (Table 3). Some sequences are listed as genus unnamed species to designate these as potentially novel sequences unmatched in the data base. No specific pattern of predominance was seen across treatments. However, Clostridium sp., Papillibacter sp., Ruminococcus sp., and Lactobacillus $\mathrm{sp}$. were detected at relatively high numbers. There was no pattern for species relative abundance in Eimeria postinfection ceca (Table 3). Every postinfection group had at least one sequence hit at or above $10 \%$.

Potential human enteropathogens were detected at pre- and postinfection. A Campylobacter sp. was detected in all groups except UU at postinfection. Shigella boydii, S. dysenteriae, $S$. flexneri, S. sonni, and an unidentified Shigella sp. were detected. The Shigella boydii and S. flexneri were not detected at preinfection. The poultry pathogen Clostridiun perfringens was detected at postinfection in unmedicated-infected control (UI), BC30, and Crina Poultry Plus 300 ppm (CPP) (data not shown).

PCA (Fig. 1) of pyrosequence data and body weight (BW) shows the relationship among cecal MC at pre- and postinfection. Distances between samples correlate with increasing MC dissimilarity. The first axis explained $31.4 \%$ and second axis $21.9 \%$ of variation. The first axis corresponded to Eimeria infection ( $p=0.001)$, revealing two groups: preinfection (circles) and postinfection (squares). Significant correlation was observed between $\mathrm{MC}$ composition and the relative percent average $\mathrm{BW}\left(p=0.0001, R^{2}=0.9\right)$.

\section{Denaturing gradient gel electrophoresis}

The ileal DGGE MC dendrogram shows two main groups composed mainly of pre- and postinfection $\mathrm{MC}$, respectively (Fig. 2). However, postinfection UU clustered with the preinfection $\mathrm{MC}$ and exhibiting $78.9 \% \mathrm{SC}$ with negative control (NC) and Calsporin. The NC and Calsporin MC shared $87.9 \%$ SC, whereas UI clustered with postinfection groups. The preinfection UI exhibited very close alignment with the postinfection NC, positive control (PC), UI, and BC30.

Cecal MC also segregated into mainly pre- and postinfection groups (Fig. 3). Postinfection UU clustered with preinfection $\mathrm{MC}$ and in close association with preinfection UI $(91.8 \%)$ and UI (88.2\%). The preinfection CPF clustered with the postinfection CPP $(83.6 \%)$, whereas the postinfection CPF and UI exhibited MC most unlike the other groups (51.6\% and $54.6 \%$, respectively).

Table 3. Pyrosequenced Percentage Bacterial Species in Cecal Contents of Broilers 7 Days After Mixed Eimeria spp. Infection

\begin{tabular}{|c|c|c|c|c|c|c|c|c|}
\hline \multirow[b]{2}{*}{ Species } & \multicolumn{8}{|c|}{ Treatment } \\
\hline & UU & UI & $P C$ & $N C$ & $B C 30$ & $C A L$ & $C P P$ & $C P F$ \\
\hline Anaerofilum sp. & 0.3 & nd & 0.6 & 3.0 & 1.0 & 16.3 & 0.2 & 0.1 \\
\hline Bacteroides sp. & 8.0 & 11.6 & 23.0 & 0.2 & 6.1 & 5.7 & 15.4 & 5.7 \\
\hline Campylobacter sp. & nd & 1.6 & 0.9 & 11.9 & 1.5 & 0.4 & 1.3 & 4.2 \\
\hline Clostridium lactatifermentans & 0.8 & 10.5 & 3.1 & 0.1 & 1.7 & 1.5 & 7.5 & 0.8 \\
\hline Enterococcus sp. & nd & 0.7 & 21.4 & nd & 0.2 & 0.1 & 22.5 & 0.5 \\
\hline Escherichia coli & 0.1 & 14.9 & 1.2 & 0.4 & 6.4 & 4.6 & 1.5 & 2.1 \\
\hline Escherichia sp. & nd & 16.1 & 1.0 & 0.4 & 6.2 & 5.7 & 1.1 & 1.2 \\
\hline Faecalibacterium sp. & 14.0 & 0.6 & 1.9 & 0.5 & 2.7 & 5.2 & 3.2 & 0.8 \\
\hline Lactobacillus sp. & 1.3 & 1.1 & 0.8 & 6.7 & 16.8 & 7.1 & 0.5 & 67.3 \\
\hline Papillibacter sp. & 11.2 & 3.3 & 8.6 & 4.0 & 4.0 & 6.7 & 6.0 & 2.8 \\
\hline Ruminococcus sp. & 7.4 & 1.0 & 3.9 & 4.5 & 11.5 & 4.4 & 6.5 & 0.6 \\
\hline Shigella sonnei & nd & 1.3 & 0.1 & nd & 1.1 & 0.1 & 0.3 & 1.0 \\
\hline Shigella sp. & 0.1 & 13.5 & 1.2 & 0.2 & 4.9 & 3.6 & 1.5 & 2.5 \\
\hline Subdoligranulum sp. & 4.0 & 2.1 & 4.6 & 56.4 & 7.6 & 17.2 & 2.3 & 0.5 \\
\hline Percentage total & 47.1 & 78.3 & 72.3 & 88.2 & 71.7 & 78.7 & 69.9 & 90.1 \\
\hline
\end{tabular}




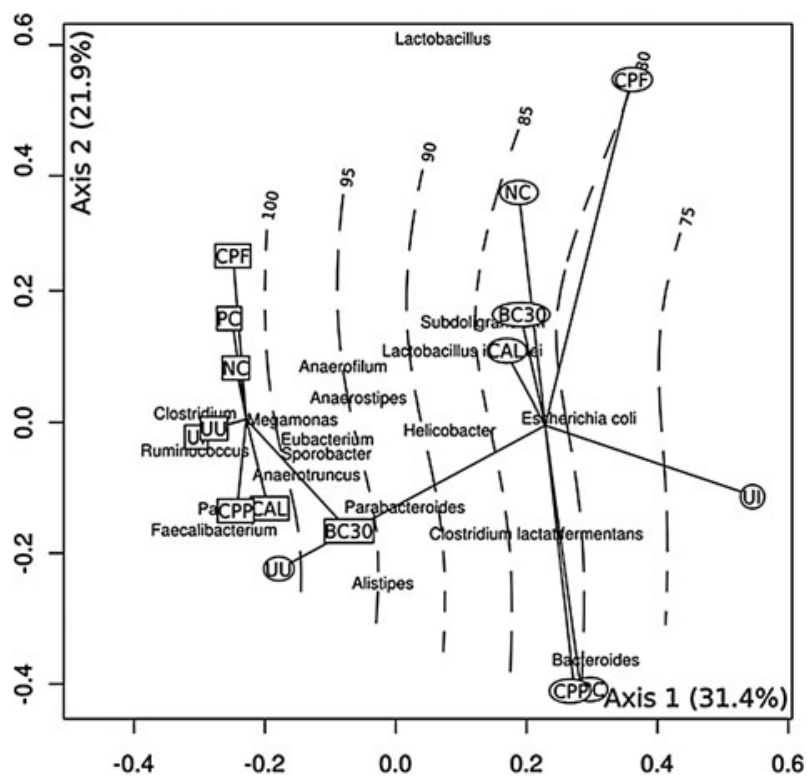

FIG. 1. Principal component analysis ordination plot of cecal bacterial microflora identified in pre- (squares) and post-Eimeria (circles) infection ceca. Solid lines connect samples into pre- and postinfection groups (centroids). Axes 1 and 2 explain $53.3 \%$ of the variation present in the data. Contour lines represent the relative percent average body weight data fit to samples ordination scores. Taxa names indicate the most abundant taxa occuring at different locations in ordination space. The difference between the groups (centroids) is significant $(p<0.001)$. Abbreviations inside the symbols represent treatments as indicated in Table 1.

PCA revealed that Eimeria infection significantly $(p<0.009)$ affected ileal MC (Fig. 4). Similar results were seen in the PCA of cecal MC (Fig. 5). The arrangement of groups shows that cecal MC at preinfection have significantly $(p<0.04)$ different MC compared to postinfection broilers.

\section{Growth and feed performance}

No real differences in treatments for body weight gain (BWG) or feed conversion ratio (FCR) were seen in broilers at
14 days of age (Table 4). By 22 days, group UU were significantly $(p<0.05)$ more BWG than all other treatment groups, with the lowest BWG in UI, broilers given no treatment other than infection. The UU group also had better $(p<0.05)$ FCR than UI, NC, and CPP and with a ratio numerically lower than all other groups. Broilers in UI, accordingly, had the worst FCR of all treatment groups.

\section{Discussion}

An objective of the current line of poultry MC studies is to identify bio-organisms and chemical agents that enhance production and off-set negative effects of pathogen colonization or stress conditions. Treatment effects in the present study seen as differences in DGGE banding patterns likely resulted from bactericidal, bacteriostatic, or beneficial effects of the probiotic Bacillus spp. or bioactive EO. The current study, to our knowledge, is one of the first to combine a broadspectrum species molecular identification, with far less bacterial selection than traditional culturing, and the DGGE visual referencing. However, DGGE band identity is still dependent upon imprecise excision of closely positioned gel bands for PCR and database referencing. Alternatively, pyrosequencing per sample costs make DGGE more attractive as a treatment discriminating tool for MC studies.

Broiler digestive $\mathrm{MC}$ were influenced by two commercial probiotics and two EO. From pre- to postinfection, there was an increase in the number of species at $1 \%$ or more. This increase may be the result of broiler and MC maturation ( $\mathrm{Lu}$ et al., 2003) as well as from treatments. However, DGGE demonstrated the impact of Eimeria infection on MC as seen with the clustering of postinfection UU with preinfection groups. Additionally, PCA contour lines, showing relative percent average BW, along with positioning of postinfection UU with preinfection groups, indicated the pronounced effect of infection on BW loss as well as shifts in MC.

Lactobacillus, Enterococcus, and Es. coli were detected at relatively high levels and are common commercial probiotic ingredients (Huys et al., 2006). In general, in vitro and in vivo oregano EO and BMD treatment reduce the numbers of these genera (Kivanc et al., 1991; Elgayyar et al., 2001; Burt and Reinders, 2003; Peñalver et al., 2005; Biggs and Parsons, 2008).
FIG. 2. Dendrogram of denaturing gradient gel electrophoresis comparing $16 \mathrm{~S}$ rDNA amplicon patterns of ileal (Il) contents bacterial populations in chicks at 14 days of age pre-infection and at 22 days of age and 7 days after mixed Eimeria spp. postinfection. Relative similarity of band patterns is indicated by their grouping on the dendogram and the percentage similarity coefficient (bar). Abbreviations represent treatments as indicated in Table 1.

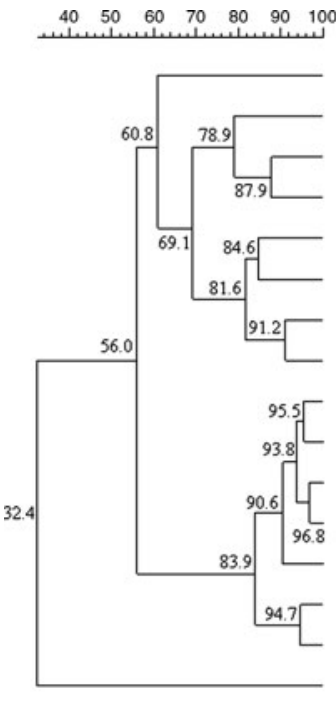

IIUU Pre IIUU Post IlNC Pre IlBC30 Pre IIPC Pre IlCPF Pre IlCAL Pre IlCPP Pre IlPC Post IINC Post IlUI Post IlBC30 Post IIUI Pre IlCPP Post IlCPF Post IlCA Post 


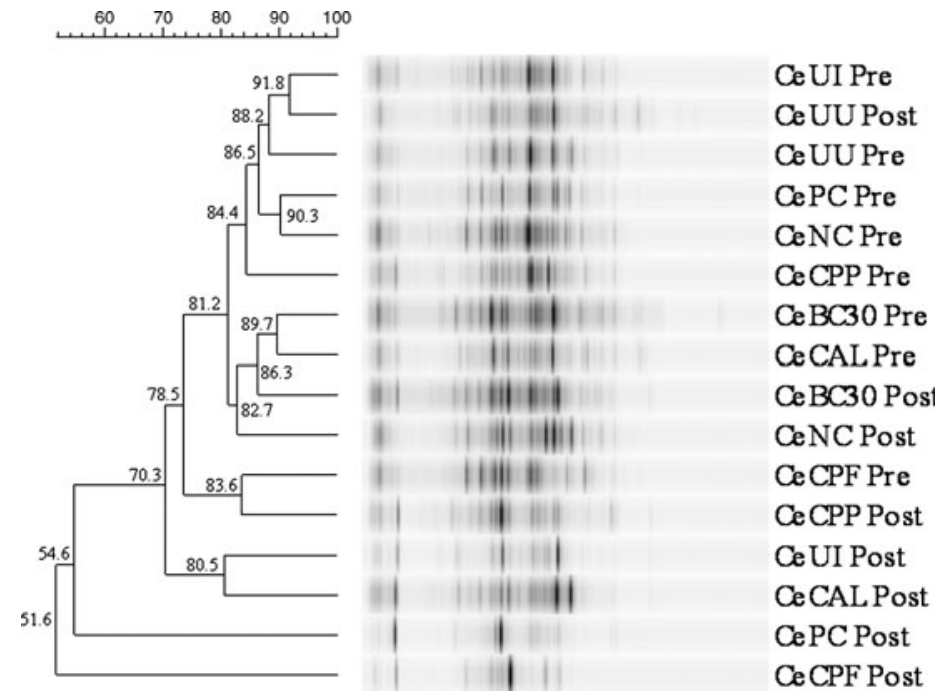

FIG. 3. Dendrogram of denaturing gradient gel electrophoresis comparing $16 \mathrm{~S}$ rDNA amplicon patterns of cecal $(\mathrm{Ce})$ contents bacterial populations in chicks at 14 days of age pre-infection and at 22 days of age and 7 days after mixed Eimeria spp. postinfection. Relative similarity of band patterns is indicated by their grouping on the dendogram and the percentage similarity coefficient (bar). Abbreviations represent treatments as indicated in Table 1.
Cecal Lactobacillus at preinfection was relatively high in CPP and $\mathrm{CPF}$, whereas Lactobacillus was high in $\mathrm{BC} 30$ and $\mathrm{CPF}$ at postinfection. The lack of apparent inhibition of Lactobacillus at pre- and postinfection or Enterococcus in postinfection CPP may be due to differences in $\mathrm{EO}$ composition used in the current study as compared to others.

The commercial probiotics contained known Bacillus spp. However, an unidentified Bacillus sp. was detected below 1\% in postinfection $\mathrm{UU}, \mathrm{BC} 30$, and $\mathrm{CPF}$ and did not register as a Bacillus in the probiotics administered. This result is not surprising, since residence of introduced probiotic bacteria is often transient and at undetectable levels (Freter et al., 1983; Jin et al., 1998; Priyankarage et al., 2004).

In spite of the application of commercial probiotics and the presence of commonly applied probiotic bacteria, several potential human enteropathogens were detected, mainly at Eimeria postinfection. However, these enteropathogens were not detected or were at low numbers in UU broilers. Eimeria oocyte vaccination in 1-day-old broilers can render immune protection against Salmonella (Volkova et al., 2011). However, Eimeria infection in 2-week-old broilers can disrupt cecal MC (Hume et al., 2006; Oviedo-Rondón et al., 2006). This disruption appears sufficient to render broilers vulnerable to enteropathogen colonization. A low level Campylobacter colonization was seen at postinfection, but was not detected in UU and was above $10 \%$ in NC (given Coban). Usually, there is no association between coccidiostat treatment and increased susceptibility to enteropahtogen colonization.

While pyrosequencing is more useful at detecting minor changes in specific taxa, DGGE gives a qualitative profile of MC with sensitivity at $1 \%$ of the total community. Ileal and cecal DGGE profiles confirmed previous reports demonstrating the effects of Eimeria on MC (Hume et al., 2006; Oviedo-Rondón et al., 2006). Ileal MC separated into two main groups, pre- and postinfection. This partitioning is partially an expected result of broiler aging. Grouping of postinfected ileal UU with preinfected groups reflects that this group received no medication and no infection. Apparently, aging did not have as much effect on ileal MC as did treatments and Eimeria infection. However, preinfected UI was more similar to post- than to preinfected broilers. Visual examination of band patterns suggests that the main difference was not the band presence or absence, but, possibly, relative band intensity. Differences in intensities may reflect minor and natural variation in aging populations (Zhu et al., 2002; Hume et al., 2003). The greater similarity between postinfection than between preinfection groups suggests stabilization of MC with age and lessening of variation as MC mature. Of course, another consideration may be substantial influence exerted by Eimeria.

There were some similarities in cecal MC clustering as seen in ilea. Cecal postinfection UU profile was very similar to preinfection UU and UI. Grouping of cecal UU with UI is more likely than the same occurring in ilea due to the more

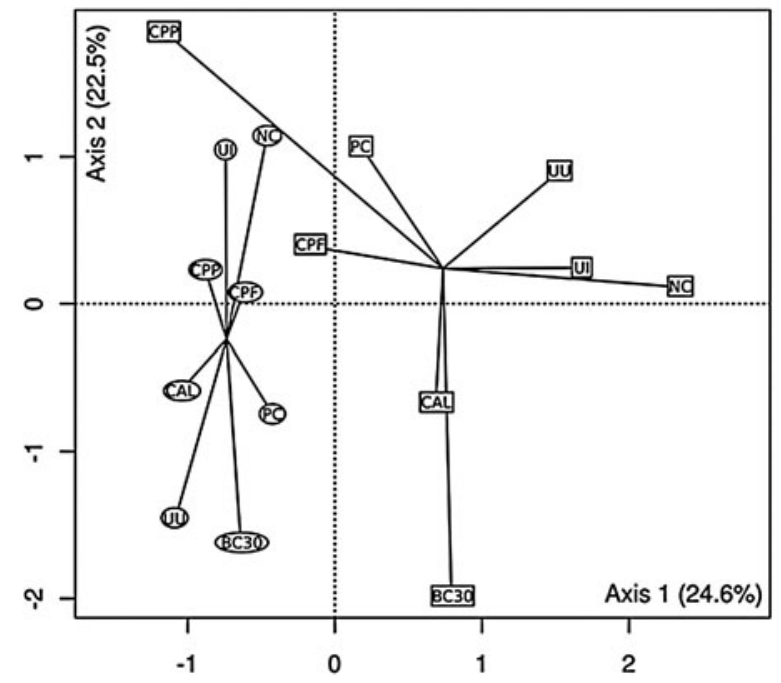

FIG. 4. Principal component analysis ordination plot of denaturing gradient gel electrophoresis profiles from pre(squares) and post-Eimeria (circles) infection ceca. Solid lines connect samples into pre- and postinfection groups (centroids). Axes 1 and 2 explain $47.1 \%$ of the variation present in the data. The difference in centroids is significant $(p<0.009)$. A inside the symbols represent treatments as indicated in Table 1. 


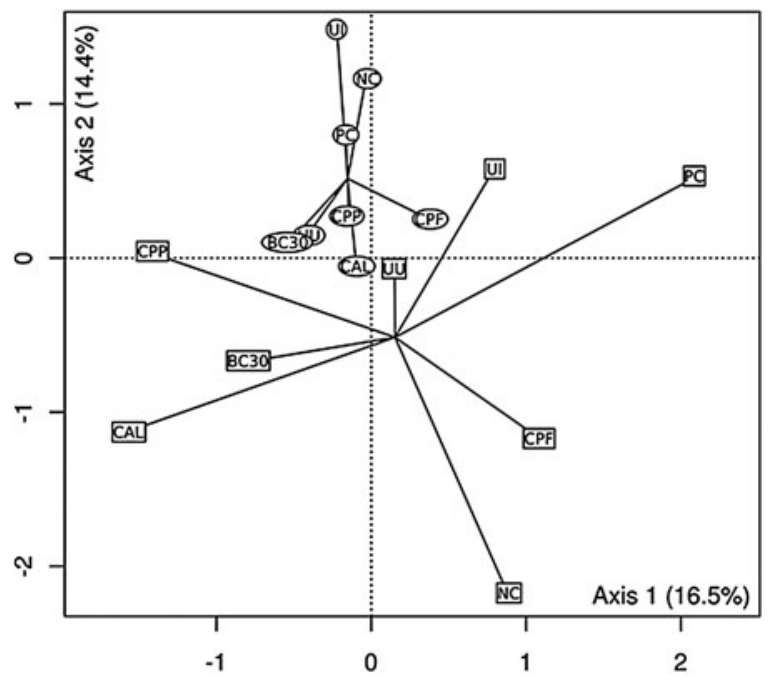

FIG. 5. Principal component analysis ordination plot of denaturing gradient gel electrophoresis profiles from pre(squares) and post-Eimeria (circles) infection ilea. Solid lines connect samples into pre- and postinfection groups (centroids). Axes 1 and 2 explain $30.9 \%$ of the variation present in the data. The difference in centroids is significant $(p<0.04)$. Abbreviations inside the symbols represent treatments as indicated in Table 1.

stable, uniform cecal environment. Similar to what was seen in postinfection ilea, preinfection PC and NC, while grouped with UU and UI, reflect the lack of treatment with probiotic or specific EO on these populations.

Intestinal MC are vital to broiler performance and health. Eimeria infection effects on MC may be seen as changes in
BWG and feed efficiency. At postinfection, BWG except in UU reflect the negative effects of infection. Poor performance is an expected result of Eimeria infection (Moynihan, 1950) and multiple approaches target conventional antimicrobials to reduce these negative effects. By day 22 in the current study, none of the feed additives reduced the negative effects of infection on BWG to levels seen in UU. However, all feed additives had some positive effect on decreased weight gain. Eimeria infection alters the MC in affected broilers (Hume et al., 2006; Oviedo-Rondón et al., 2006). The inference is that the debilitating effects of Eimeria infection were lessened by these fed additives. The ultimate goal is to identify specific effects of feed additives on beneficial and debilitating MC and to identify those MC components most supportive of broiler production.

Treatments including the two commercial probiotics and the two specific EO modified broiler ileal and cecal digestive MC. Pyrosequencing was very sensitive at detecting shifts in individual identified sequences, whereas DGGE was adept at detecting gross shifts in entire populations. These combined techniques offered added versatility toward unraveling the effects of Eimeria infection and performance enhancing feed additives on broiler MC. Results show modifications of MC resulting from treatments before infection with a standard mixed Eimeria spp. as well as the effects after mild infection. Pyrosequencing and DGGE offer individual advantages in addition to their respective sensitivity and ability to detect gross shifts in populations. The effects of infection and treatment with either performance enhancing or therapeutic antibiotics are well understood at the macro level. Efforts to uncover the contributions made by shifts in individual bacteria in the greater community, similarly as applied in the current study, will aid in the development of intervention strategies for protection and treatment against pathogen

Table 4. Average Body Weight Gain and Feed Conversion Ratio of Broilers Fed Diets with Different Treatments in the Preinfection Period (1-14 Days of Age) in Floor Pens with Used Litter, and the Period of Infection (15-22 Days of Age), with Mixed Eimeria spp., in Cages

\begin{tabular}{|c|c|c|c|c|c|c|c|c|}
\hline \multirow[b]{2}{*}{ Variables } & \multicolumn{8}{|c|}{ Treatments } \\
\hline & UU & UI & $P C$ & $N C$ & BC30 & $C A L$ & $C P P$ & $C P F$ \\
\hline \multicolumn{9}{|l|}{ 1-14 days } \\
\hline BWG (kg) & 0.389 & 0.389 & 0.394 & 0.396 & 0.396 & 0.381 & 0.402 & 0.381 \\
\hline FCR $(\mathrm{kg} / \mathrm{kg})$ & $1.502^{\mathrm{ab}}$ & $1.579^{\mathrm{a}}$ & $1.475^{\mathrm{ab}}$ & $1.512^{\mathrm{ab}}$ & $1.437^{\mathrm{ab}}$ & $1.466^{\mathrm{ab}}$ & $1.381^{\mathrm{b}}$ & $1.485^{\mathrm{ab}}$ \\
\hline \multicolumn{9}{|l|}{15 to 22 days } \\
\hline BWG (kg) & $0.556^{\mathrm{a}}$ & $0.251^{\mathrm{c}}$ & $0.372^{\mathrm{b}}$ & $0.367^{\mathrm{b}}$ & $0.387^{\mathrm{b}}$ & $0.315^{\mathrm{bc}}$ & $0.318^{\mathrm{b}}$ & $0.369^{\mathrm{b}}$ \\
\hline \multirow[t]{3}{*}{ FCR (kg/kg) } & $1.136^{\mathrm{c}}$ & $1.638^{\mathrm{a}}$ & $1.245^{\mathrm{bc}}$ & $1.368^{\mathrm{b}}$ & $1.334^{\mathrm{bc}}$ & $1.246^{\mathrm{bc}}$ & $1.348^{\mathrm{b}}$ & $1.325^{\mathrm{bc}}$ \\
\hline & \multicolumn{8}{|c|}{ Statistics } \\
\hline & SEM & \multicolumn{2}{|c|}{ p-Value } & $C V(\%)$ & & & & \\
\hline \multicolumn{9}{|l|}{ 1-14 days } \\
\hline BWG (kg) & 0.008 & \multicolumn{2}{|c|}{0.665} & 4.48 & & & & \\
\hline FCR $(\mathrm{kg} / \mathrm{kg})$ & 0.035 & \multicolumn{2}{|c|}{0.037} & 4.23 & & & & \\
\hline \multicolumn{9}{|l|}{ 15-22 days } \\
\hline BWG (kg) & 0.018 & \multicolumn{2}{|c|}{$<0.0001$} & 10.70 & & & & \\
\hline FCR $(\mathrm{kg} / \mathrm{kg})$ & 0.046 & \multicolumn{2}{|c|}{$<0.0001$} & 7.80 & & & & \\
\hline
\end{tabular}

All probiotic and essential oil treatments contained the same ionophore as PC and NC.

$\mathrm{a}, \mathrm{b}, \mathrm{c}$ Means in rows with no common superscript differ significantly $(p<0.05)$ by Tukey's test.

PC, positive control (antibiotic + ionophore); NC, negative control (ionophore only); CPP, Crina Poultry Plus, essential oil at 300 ppm; CPF, Crina Poultry AF, essential oil at 100 ppm; BWG, body weight gain; FCR, feed conversion ratio; CV, coefficient of variation. 
infection and colonization. Molecular techniques such as pyrosequencing, in contrast to the more bio-selective traditional culture techniques, offer a considerably more objective view of minor and major contributors to the general MC.

\section{Disclaimer}

Mention of trade names or commercial products in this article is solely for the purpose of providing specific information and does not imply recommendation or endorsement by the U.S. Department of Agriculture.

\section{Disclosure Statement}

No competing financial interests exist.

\section{References}

Andreotti R, Perez de Leon AA, Dowd SE, et al. Assessment of bacterial diversity in the cattle tick Rhipicephalus (Boophilus) microplus through tag-encoded pyrosequencing. BMC Microbiol 2011;11:6.

Bailey MT, Dowd SE, Parry NM, et al. Stressor exposure disrupts commensal microbial populations in the intestines and leads to increased colonization by Citrobacter rodentium. Infect Immun 2010a;78:1509-1519.

Bailey MT, Walton JC, Dowd SE, et al. Photoperiod modulates gut bacteria composition in male Siberian hamsters (Phodopus sungorus). Brain Behav Immun 2010b;24:577-584.

Barnes EM, Impey CS, and Coope DM. Competitive exclusion of salmonellas from the newly hatched chick. Vet Rec 1980;106:61.

Biggs P and Parsons CM. The effects of Grobiotic-P on growth performance, nutrient digestibilities, and cecal microbial populations in young chicks. Poult Sci 2008;87:1796-1803.

Blankenship LC, Bailey JS, Cox NA, et al. Two-step mucosal competitive exclusion flora treatment to diminish salmonellae in commercial broiler chickens. Poult Sci 1993;72:1667-1672.

Burt SA and Reinders RD. Antibacterial activity of selected plant essential oils against Escherichia coli O157:H7. Lett Appl Microbiol 2003;36:162-167.

Callaway TR, Dowd SE, Edrington TS, et al. Evaluation of the bacterial diversity in the rumen and feces of cattle fed diets containing levels of dried distiller's grains plus solubles using bacterial tag-encoded FLX amplicon pyrosequencing (bTEFAP). J Anim Sci 2010;88:3977-3983.

Chessel D, Dufour A-B, and Thioulouse J. The ade4 package. I. One-table methods. RNews 2004;4:5-10.

Cole JR, Wang Q, Cardenas E, et al. The ribosomal database project: improved alignments and new tools for rRNA analysis. Nucleic Acids Res 2009;37:D141-145.

Dibner JJ and Richards JD. Antibiotic growth promoters in agriculture: history and mode of action. Poult Sci 2005;84:634643.

Dowd SE, Zaragoza J, Rodriguez JR, et al. Windows .NET network distributed basic local alignment search toolkit (W.NDBLAST). BMC Bioinform 2005;6:93.

Elgayyar M, Draughon FA, Golden DA, et al. Antimicrobial activity of essential oils from plants against selected pathogenic and saprophytic microorganisms. J Food Prot 2001;64:10191024.

Finegold SM, Dowd SE, Gontcharova V, et al. Pyrosequencing study of fecal microflora of autistic and control children. Anaerobe 2010;16:444-453.
Freter R, Brickner H, Fekete J, et al. Survival and implantation of Escherichia coli in the intestinal tract. Infect Immun 1983;39:686-703.

Flint JF and Garner MR. Feeding beneficial bacteria: a natural solution for increasing efficiency and decreasing pathogens in animal agriculture. J Appl Poult Res 2009;18:367-378.

Gontcharova V, Youn E, Sun Y, et al. A comparison of bacterial composition in diabetic ulcers and contralateral intact skin. Open Microbiol J 2010a;4:8-19.

Gontcharova V, Youn E, Wolcott RD, et al. Black box chimera check (B2C2): a Windows-based software for batch depletion of chimeras from bacterial 16S rRNA gene datasets. Open Microbiol J 2010b;4:6.

Handl S, Dowd SE, Garcis-Mazcorro JF, et al. Massive parallel $16 \mathrm{~S}$ rRNA gene pyrosequencing reveals highly diverse fecal bacterial and fungal communities in healthy dogs and cats. FEMS Microbiol Ecol 2011;76:301-310.

Hume ME, Kubena LF, Edrington TS, et al. Poultry digestive microflora biodiversity as indicated by denaturing gradient gel electrophoresis. Poult Sci 2003;82:1100-1107.

Hume ME, Oviedo-Rondón EO, and Clemente-Hernández S. Effects of feed additives and mixed Eimeria spp. infection on intestinal microbial ecology of broilers. Poult Sci 2006;85:21062111.

Huys G, Vancanney M, D'Haene K, et al. Accuracy of species identity of commercial bacterial cultures intended for probiotic or nutritional use. Res Microbiol 2006;1570:803-810.

Ishak HD, Plowes R, Sen R, et al. Bacterial diversity in Solenopsis invicta and Solenopsis geminata ant colonies characterized by $16 S$ amplicon 454 pyrosequencing. Microb Ecol 2011;61:821831.

Jamroz D, Wiliczkiewicz A, Wertelecki T, et al. Use of active substances of plant origin in chicken diets based on maize and locally grown cereals. Br Poult Sci 2005;46:485-493.

Jin LZ, Ho YW, Abdullah N, et al. Growth performance, intestinal microbial populations, and serum cholesterol of broilers fed diets containing Lactobacillus cultures. Poult Sci 1998; 77:1259-1265.

Jukes TH. Antibiotics in Nutrition. New York: Medical Encyclopedia, Inc., 1955.

Jukes TH, Stokstad ELR, Taylor RR, et al. Growth-promoting effect of aureomycin on pigs. Arch Biochem 1950;26:324-325.

Jung SJ, Houde R, Baurhoo B, et al. Effects of galacto-oligosaccharides and a Bifidobacteria lactis-based probiotic strain on the growth performance and fecal microflora of broiler chickens. Poult Sci 2008;7:1694-1699.

Kim J, Marshall MR, and Wei C. Antibacterial activity of some essential oils components against five foodborne pathogens. J Agric Food Chem 1995;43:2839-2845.

Kivanc M, Akgul A, and Dogan A. Inhibitory and stimulatory effects of cumin, oregano and their essential oils on growth and acid production of Lactobacillus plantarum and Leuconostoc mesenteroides. Int J Food Microbiol 1991;13:81-85.

Lloyd A, Cumming R, and Kent R. Prevention of Salmonella typhimurium infection in poultry by pretreatment of chickens and poults with intestinal extracts. Aust Vet J 1977;53:82-86.

$\mathrm{Lu}$ J, Idris U, Harmon B, et al. Diversity and succession of the intestinal bacterial community of the maturing broiler chicken. Appl Environ Microbiol 2003;69:6816-6824.

Moynihan IW. The role of the protozoan parasite Eimeria acervulina in disease of the domestic chicken. Can J Comp Med Vet Sci 1950;14:74-82.

Muyzer G, De Waal EC, and Litterlinden AG. Profiling of complex microbial populations by denaturing gradient gel 
electrophoresis analysis of polymerase-chain reaction-amplified genes coding for 16S rRNA. Appl Environ Microbiol 1993;59:695-700.

[NRC] National Research Council. Nutrient Requirements of Poultry, 9th edition (revised). Washington, DC: National Academy Press, 1994.

Nisbet DJ, Corrier DE, and DeLoach JD. Effect of mixed cecal microflora maintained in continuous culture and of dietary lactose on Salmonella typhimurium colonization in broiler chicks. Avian Dis 1993;37:528-535.

Nurmi E and Rantala M. New aspects of Salmonella infection in broiler production. Nature 1973;241:210-211.

Oksanen J, Kindt R, Legendre $\mathrm{P}$, et al. Community ecology package, Version 1.17. Available at http://cran.r-project.org/ web/packages/vegan/vegan.pdf, accessed November, 2010. (Online.)

Olafson PU, Lohmeyer KH, and Dowd SE. Analysis of expressed sequence tags from a significant livestock pest, the stable fly (Stomoxys calcitrans), identifies transcripts with a putative role in chemosensation and sex determination. Arch Insect Biochem Physiol 2010;74:179-204.

Oviedo-Rondón EO, Hume ME, Hernandez C, et al. Intestinal microbial ecology of broilers vaccinated and challenged with mixed Eimeria spp., and supplemented with essential oil blends. Poult Sci 2006;85:854-860.

Peñalver $P$, Huerta $B$, Borge $C$, et al. Antimicrobial activity of five essential oils against origin strains of the Enterobacteriaceae family. APMIS 2005;113:1-6.

Pitta DW, Pinchak E, Dowd SE, et al. Rumen bacterial diversity dynamics associated with changing from bermudagrass hay to grazed winter wheat diets. Microb Ecol 2010;59:511-522.

Priyankarage N, Silva SSP, Gunaratne SP, et al. Efficacy of probiotics and their effects on performance, carcase characteristics, intestinal microflora and Salmonella incidence in broilers. Br Poult Sci 2004;44:S26-S27.

Schneitz C, Kiickinen T, Toivonen V, et al. Effect of BROILACT on the physicochemical conditions and nutrient digestibility in the gastrointestinal tract of broilers. Poult Sci 1998;77:426-432.
Si W, Gong J, Tsao G, et al. Antimicrobial activity of essential oils and structurally related synthetic food additives towards selected pathogenic and beneficial gut bacteria. J Appl Microbiol 2006;100:296-305.

Smith DM, Snow DE, Rees E, et al. Evaluation of the bacterial diversity of pressure ulcers using bTEFAP pyrosequencing. BMC Med Genomics 2010;3:41.

Stephenson MF, Mfuna L, Dowd SE, et al. Molecular characterization of the polymicrobial flora in chronic rhinosinusitis. J Otolaryngol Head Neck Surg 2010;39:182-187.

Volkova VV, Wills RW, Hubbard SA, et al. Associations between vaccinations against protozoal and viral infections and Salmonella in broiler flocks. Epidemiol Infect 2011;139: 206-215.

Williams WL, Tedeschi LO, Kononoff PJ, et al. Evaluation of in vitro gas production and rumen bacterial populations fermenting corn milling (co)products. J Dairy Sci 2010;93:47354743.

Zhou H, Zhou H, Gong J, et al. Appropriate chicken sample size for identifying the composition of broiler intestinal microbiota affected by dietary antibiotics, using the polymerase chain reaction-denaturing gradient gel electrophoresis technique. Poult Sci 2007;86:2541-2549.

Zhu XY, Zhong T, Pandya Y, et al. $16 \mathrm{~S}$ rRNA-based analysis of microbiota from the caecum of broiler chickens. Appl Environ Microbiol 2002;68:124-137.

Address correspondence to: Michael E. Hume, B.S., M.S., Ph.D. Food and Feed Safety Research Unit Southern Plains Agricultural Research Center Agricultural Research Service U.S. Department of Agriculture 2881 FEB Road College Station, TX 77845

E-mail: michael.hume@ars.usda.gov 
